International Journal of Advanced Chemistry, 8(1)(2020) 160-169
International Journal of Advanced Chemistry
SPC
Website: www.sciencepubco.com/index.php/IJAC
Research paper

\title{
High performance liquid chromatography quality control
}

\author{
M. E. Ghanjaoui ${ }^{1}{ }^{*}$, A. Mandil ${ }^{2}$, A. Ait Sidi Mou ${ }^{1}$, R. Slimani ${ }^{3}$ \\ ${ }^{1}$ Laboratory of Electrochemistry and Chemical Physics, Faculty of Sciences and Technologies, Mohammedia (FSTM) 28830, , Morocco \\ ${ }^{2}$ Laboratoire de Génie des Procédés et Environnement (LGPE) Casablanca, Faculty of Sciences and Technologies of Mohammedia \\ (FSTM) 28830, Mohammedia, Morocco \\ ${ }^{3}$ CERNE2D, Laboratory of Spectroscopy, Molecular Modeling, Materials \& Environment, University Mohamed V of Rabat, Morocco \\ *Corresponding author E-mail: ghanjaoui2000@hotmail.com
}

\begin{abstract}
This review gives a general overview of High Performance Liquid Chromatography (HPLC) Method for the detection, the separation and the quantification of the active compounds from the organics matrices. A brief description of the instrumentation and the method development is provided. The principles of HPLC including different separation modes and detection methods for the quantitative analysis are summarized. Finely, the validation procedures in real samples are also described.
\end{abstract}

Keywords: HPLC; Analytical method; Validation; Development; Quality Control.

\section{Introduction}

Liquid chromatography was initially discovered as an analytical technique in the early twentieth century and was first used as a method of separating colored compounds. This is where the name chromatography chroma means color, graphy means writing, was derived. A Russian botanist named Mikhail S. Tswett used a rudimentary form of chromatographic separation to purify mixtures of plant pigments into the pure constituents. He separated the pigments based on their interaction with a stationary phase, which is essential to any chromatographic separation. The stationary phase he used was powdered chalk and aluminia, the mobile phase in his separation was the solvent [1].

High-performance liquid chromatography (or High pressure liquid chromatography, HPLC) is a technique in analytical chemistry used to separate, identify, and quantify each component in a mixture [2].

The enormous success of HPLC can be attributed to a number of inherent features associated with reproducibility, ease of selectivity manipulation, and generally high recoveries. The most significant feature is the excellent resolution that can be achieved under a wide range of conditions for very closely related molecules, as well as structurally quite distinct molecules [3], [4].

The principle is that a solution of the sample is injected into a column of a porous material (stationary phase) and a liquid (mobile phase) is pumped at high pressure through the column. The separation of sample is based on the differences in the rates of migration through the column arising from different partition of the sample between the stationary and mobile phase. Depending upon the partition behaviour of different components, elution at different time takes place. The sample compound with the greater affinity to the stationary layer will travel slower and for a shorter distance in comparison to compounds with less affinity which travel faster and for a longer distance [5]. Generally, the detector attached to the HPLC unit generates ultraviolet rays of particularly specified wavelength and the eluting sample is subjected to these ultraviolet rays. Molecules of the eluting species get excited by absorbing the energy of the ultraviolet rays, during the process of de-excitation energy is released which is being recorded by the detector [6], [7].

A signal proportional to the energy change is generated and recorded in the form of a graph called chromatogram. The absorption of the ultraviolet rays depends on the functional group of the eluting species, because every functional group requires a particular amount of energy for its excitation, which is carried by ultraviolet light of a certain wavelength. However, sometimes specifically reported costly apparatus is often not available in the research labs. To achieve the desired analysis on the available set of apparatus, certain parameters need to be tuned, this is called HPLC method development. The main goals of this review are to describing the instrumentation of the method, to explaining all parameters that influence the HPLC results and summarizing the validation procedures of developed method.

\section{Different types of HPLC}

There are following variants of HPLC, depending upon the phase system (stationary) in the process:

\subsection{Normal phase HPLC}


This method separates analytes on the basis of polarity. NP-HPLC uses polar stationary phase and non-polar mobile phase. Therefore, the stationary phase is usually silica and typical mobile phases are hexane, methylene chloride, chloroform, diethyl ether, and mixtures of these.

Polar samples are thus retained on the polar surface of the column packing longer than less polar materials [8].

\subsection{Reversed phase chromatography}

Reversed phase HPLC (RP-HPLC or RPC) has a non-polar stationary phase and an aqueous, moderately polar mobile phase. RPC operates on the principle of hydrophobic interactions, which result from repulsive forces between a polar eluent, the relatively non-polar analyte, and the non-polar stationary phase. The binding of the analyte to the stationary phase is proportional to the contact surface area around the non-polar segment of the analyte molecule upon association with the ligand in the aqueous eluent [9].

\subsection{Ion exchange chromatography}

In Ion-exchange chromatography, retention is based on the attraction between solute ions and charged sites bound to the stationary phase. Ions of the same charge are excluded. This form of chromatography is widely used in purifying water, Ligand-exchange chromatography, Ion-exchange chromatography of proteins, High-pH anion-exchange chromatography of carbohydrates and oligosaccharides, etc [10].

\subsection{Size exclusion chromatography}

Size exclusion chromatography (SEC), also called as gel permeation chromatography or gel filtration chromatography mainly separates particles on the basis of size. It is also useful for determining the tertiary structure and quaternary structure of proteins and amino acids. This technique is widely used for the molecular weight determination of polysaccharides [11].

\subsection{Bio-affinity chromatography}

Separation based on specific reversible interaction of proteins with ligands. Ligands are covalently attached to solid support on a bioaffinity matrix, retains proteins with interaction to the column-bound ligands.

Proteins bound to a bioaffinity column can be eluted in two ways:

Biospecific elution: inclusion of free ligand in elution buffer which competes with column bound ligand.

Aspecific elution: change in $\mathrm{pH}$, salt, etc. which weakens interaction protein with column-bound substrate.

Because of specificity of the interaction, bioaffinity chromatography can result in very high purification in a single step (10 - 1000-fold) [12].

\section{Instrumentation of HPLC}

The most important components of the HPLC instrument are: mobile phase/solvent reservoir, solvent delivery system, sample introduction device, column, detectors, data collection and output [13] (Figure 1).

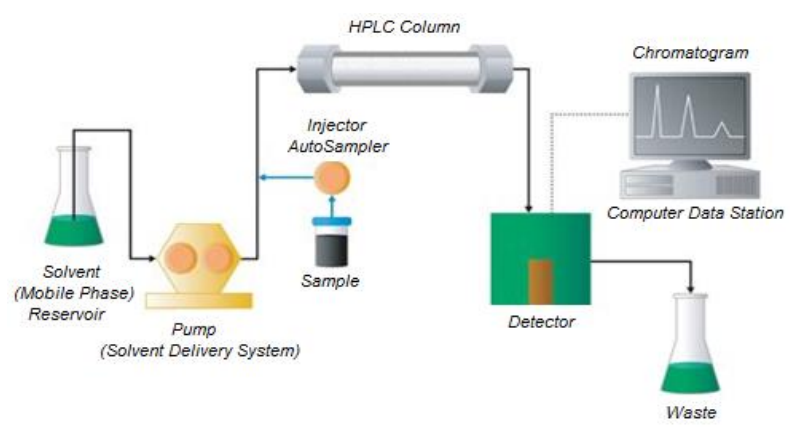

Fig. 1: High-Performance Liquid Chromatography HPLC System.

\subsection{Mobile phase/solvent reservoir}

The reservoir that holds the mobile phase is often no more than a glass bottle. Often, the reagent bottle that holds our HPLC solvent can be used as a reservoir. Solvent is delivered from the reservoir to the pump by means of Teflon tubing -- called the "inlet line" to the pump. Some HPLC systems like the Agilent 1100 shown at the right have special compartments to hold one or more mobile phase reservoirs. The reservoirs in these systems may have additional features that allow the mobile phase to be degassed and isolated from contact with air.

\subsection{Solvent delivery system}

The solvent delivery system is described like a deliver system of continuous pulse free flow of mobile phase to the HPLC regardless of the system back pressure.

\subsection{Injection of the sample}


The injection of a sample at atmospheric pressure into the system, at high pressure, represents a critical step in the chromatographic process. Sample injection valves, or switching valves, are used to introduce reproducible amounts of sample into the HPLC eluent stream without causing changes in pressure or flow.

\subsection{Column}

The column is the heart of a HPLC system, There are several types of matrices for support of the stationary phase, including silica, polymers, and alumina. Silica is the most common matrix for HPLC columns. Silica matrices are robust, easily derivatized, manufactured to consistent sphere size, and does not tend to compress under pressure. Silica is chemically stable to most organic solvents and to low $\mathrm{pH}$ systems.

\subsection{Detector}

An overview of various HPLC detectors is provided with discussion of unique detector characteristics and a comparison of advantages and drawbacks between them. Focus is placed on the most common detectors, including UV/Vis absorbance, fluorescence, electrochemical, conductivity, refractie index, and mass spectrometry detectors.

\subsection{Data collection and output}

The output is recorded as a series of peaks, each one representing a compound in the mixture passing through the detector and absorbing UV light (in the case of HPLC-UV/Vis). The area under the peak is proportional to the amount of substance, which is passed through detector, and this area can be calculated automatically by the computer linked to the display.

\section{HPLC development method}

There are many factors to consider when developing methods. The initially collect the information about the analyte's physiochemical properties ( $\mathrm{pKa}, \log \mathrm{P}$, solubility) and determining which mode of detection would be suitable for analysis. The majority of the analytical development effort goes into validating a stability indicating HPLC-method. The goal of the HPLC-method is to try \& separate quantify the main active compound, any reaction impurities, all available synthetic inter-mediates and any degradants [14].

\subsection{Physicochemical properties of the active compound}

Physicochemical properties of the active compound play an important role in method development. For method development one has to study the physical properties like solubility, polarity, $\mathrm{pKa}$ and $\mathrm{pH}$ of the molecule. Polarity is a physical property of a compound. It helps an analyst, to decide the solvent and composition of the mobile phase.

Selection of diluents is based on the solubility of analyte. The analyte must be soluble in the diluents and must not react with any of the diluent components. The diluent should match to the starting eluent composition of the assay to ensure that no peak distortion will occur, especially for early eluting components.

The acidity or basicity of a substance is defined most typically by the $\mathrm{pH}$ value. The $\mathrm{pH}$ value is defined as the negative of the logarithm to base 10 of the concentration of the hydrogen ion, $\mathrm{pH}=-\log _{10}[\mathrm{H} 3 \mathrm{O}+]$. Selecting a proper $\mathrm{pH}$ for ionizable analytes often leads to symmetrical and sharp peaks in HPLC. Sharp, symmetrical peaks are necessary in quantitative analysis in order to achieve low detection limits, low relative standard deviations between injections, and reproducible retention times.

The $\mathrm{pKa}$ is characteristic of a particular compound, and it tells how readily the compound gives up a proton.

An acid dissociation constant is a particular example of equilibrium constant. For the specific equilibrium between a monoprotic acid HA and its conjugate base $\mathrm{A}^{-}$.

It turns that the $\mathrm{pKa}$ of an acid is the $\mathrm{pH}$ at which it is exactly half dissociated. This can be shown by rearranging the expression for Ka:

$\mathrm{pH}=\mathrm{pKa}-\log _{10}([\mathrm{AH}] /[\mathrm{A}-])$

At half-neutralization the $\mathrm{pH}$ is numerically equal to $\mathrm{pKa}$. Conversely, when $\mathrm{pH}=\mathrm{pKa}$, the concentration of $\mathrm{HA}$ is equal to the concentration of $\mathrm{A}^{-}$.

The buffer region extends over the approximate range $\mathrm{pKa} \pm 2$, though buffering is weak outside the range pKa \pm 1 . At pKa \pm 1 , $\left[\mathrm{A}^{-}\right] /[\mathrm{HA}]=10$ or $1 / 10$. If the $\mathrm{pH}$ is known, the ratio may be calculated. This ratio is independent of the analytical concentration of the acid. When the $\mathrm{pKa}$ and analytical concentration of the acid are known, the extent of dissociation and $\mathrm{pH}$ of a solution of a monoprotic acid can be easily calculated [15-18].

\subsection{Column selection}

The heart of a HPLC system is the column. Changing a column will have the greatest effect on the resolution of analytes during method development. Generally, modern reverse phase HPLC columns are made by packing the column housing with spherical silica gel beads which are coated with the hydrophobic stationary phase. The stationary phase is introduced to the matrix by reacted a chlorosilane with the hydroxyl groups present on the silica gel surface.

There are several types of matrices for support of the stationary phase, including silica, polymers, and alumina. Silica is the most common matrix for HPLC columns. Silica matrices are robust, easily derivatized, manufactured to consistent sphere size, and does not tend to compress under pressure. Silica is chemically stable to most organic solvents and to low $\mathrm{pH}$ systems. One shortcoming of a silica solid support is that it will dissolve above $\mathrm{pH}$ 7. In recent years, silica supported columns have been developed for use at high $\mathrm{pH}$ [19].

\subsection{Shape and particle size effect}


Generally, Smaller particle results in a greater number of theoretical plates, or increased separation efficiency. However, the use of smaller particles also results in increased backpressure during chromatography and the column more easily becomes plugged [20].

\subsection{Common stationary phases}

The Common stationary phases are C4 (butyl), C8 (octyl), C18 (octadecyl), nitrile (cyanopropyl), and phenyl (phenyl propyl) columns. In general, longer alkyl chains, higher phase loading, and higher carbon loads provide greater retention of non-polar analytes.

Commonly used reverse phase columns and their uses are listed below. Propyl (C3), Butyl (C4), and Pentyl (C5) columns are useful for ion- pairing chromatography. Examples: include Zorbax SB-C3, YMC-Pack C4, and Luna C5. These columns are generally less stable to hydrolysis than columns with longer alkyl chain. Octyl (C8) columns have wide applicability. This phase is less retentive than the C18 phases, but is still quite useful for pharmaceuticals. Example: include (Zorbax SB-C8, Luna C8 and YMC-Pack-MOS). Octadecyl (C18, ODS) columns are the most widely used and tend to be the most retentive for non-polar analytes. Examples include Zorbax SB-C18, YMC- Pack ODS and Luna C18. Xterra RP-C18 and Zorbax Extend-C18 columns have been formulated to tolerate high $\mathrm{pH}$ systems (pH $>$ 7, normally up to $\mathrm{pH} 11$ ).

Phenyl $(\mathrm{Ph})$ columns offer unique selectivity from the alkyl phases and are generally less retentive than $\mathrm{C} 8$ or C18 phases. Phenyl columns are commonly used to resolve aromatic compounds. Examples include Zorbax SB-Phenyl, YMC-Pack Phenyl and Luna PhenylHexyl. Nitrile (CN or cyano) columns are polar and can be used for both reverse and normal phase applications. This phase is often used to increase retention of polar analytes. Examples include Zorbax SB-CN, Luna-CN, and YMC-Pack CN.

The type of column chosen for a particular separation depends on the compound and the aim of analysis [21-27].

\subsubsection{Column temperature}

Column temperature control is important for long-term method reproducibility as temperature can affect selectivity. A target temperature in the range of $30-40{ }^{\circ} \mathrm{C}$ is normally sufficient for good reproducibility. Use of elevated temperature can be advantageous for several reasons. First, operating at a temperature higher than ambient reduces the viscosity of the mobile phase and thus the overall backpressure on the column. Lower system pressures allow for faster flow rates and thus faster analyses. The temperature may also affect selectivity patterns because analytes will respond dissimilarly to different temperatures [28].

\subsection{Mobile phase solvent type}

Acetonitrile (ACN), methanol (MeOH) and tetrahydrofuran (THF) are commonly used solvents in RP-HPLC having low UV cut-off of 190, 205 and $212 \mathrm{~nm}$ respectively. These solvents are miscible with water. Mixture of acetonitrile and water is the best initial choice for the mobile phase during method development.

The mobile phase effects resolution, selectivity and efficiency. In reverse phase chromatography, the mobile phase consists of an aqueous buffer and a non-UV active water miscible organic solvent. The effect of the organic and aqueous phase and the proportions in which they are mixed will affect the analysis of the molecule.

Selection of the mobile-phase and gradient conditions is dependent on the ionogenic nature of the analyte and the hydrophobicity of the analytes in the mixture respectively. Acidic analytes in buffers of sufficiently low $\mathrm{pH}$ will remain unchanged, increasing retention. Conversely, at higher $\mathrm{pH}$ neutral basic compounds will be more retained, and ionized acidic compounds will elute earlier. Peak splitting may be observed if the pKa of a compound is similar to the pKa of the buffer, and the analyte elutes as both a charged and uncharged species. The $\mathrm{pH}$ of a buffer will not greatly affect the retention of non-ionizable sample components [29].

\subsubsection{Isocratic elution}

A separation that employs a single solvent or solvent mixture of constant composition.

\subsubsection{Gradient elution}

Here two or more solvent systems that differ significantly in polarity are employed. After elution is begun; the ratio of the solvents is varied in a programmed way, sometimes continuously and sometimes in a series of steps. Separation efficiency is greatly enhanced by gradient elution.

\subsection{Buffer selection}

Choice of buffer is typically governed by the desired $\mathrm{pH}$. It is important that the buffer has a pKa close to the desired $\mathrm{pH}$ since buffer controls $\mathrm{pH}$ best at their $\mathrm{pKa}$. A rule is to choose a buffer with a pKa value $<2$ units of the desired mobile phase $\mathrm{pH}$ (Table 1).

Table 1: HPLC Buffers, pKa Values and Useful pH Range

\begin{tabular}{lcc}
\hline Buffer & pKa & Useful pH Range \\
\hline Trifluoroacetic acid (TFA) & $<2$ & $1.5-2.5$ \\
$\mathrm{KH}_{2} \mathrm{PO}_{4} / \mathrm{K}_{2} \mathrm{PO}_{4}$ & 7.2 & $6.2-8.2$ \\
$\mathrm{KH}_{2} \mathrm{PO}_{4} /$ phosphoric acid & 2.1 & $1.1-3.1$ \\
Ammonium acetate & 4.8 & $3.8-5.8$ \\
& 9.2 & $8.2-10.2$ \\
Ammonium formate & 3.8 & $2.8-4.8$ \\
Ammonium hydroxide/ ammonia & 9.2 & $8.2-10.2$ \\
Potassium formate / formic acid & 9.2 & $8.2-10.2$ \\
Potassium Acetate/ acetic acid & 3.8 & $2.8-4.8$ \\
Borate $(\mathrm{H} 3 \mathrm{BO} / \mathrm{Na}$ B 307 10H2O) & 4.8 & $3.8-5.8$ \\
\hline
\end{tabular}

Generally, a buffer concentration of $10-50 \mathrm{mM}$ is adequate for small molecules. Generally, no more than $50 \%$ organic should be used with a buffer. This will depend on the specific buffer as well as its concentration. Phosphoric acid and its sodium or potassium salts are 
the most common buffer systems for reversed-phase HPLC. Phosphate buffers can be replaced with sulfate buffers when analyzing organophosphate compounds [30].

General considerations for buffer selection:

- TFA can degrade with time, is volatile, absorbs at low UV wavelengths.

- Phosphate is more soluble in methanol/water than in acetonitrile/water or THF/water.

- At pH greater than 7, phosphate buffer accelerates the dissolution of silica and severely shortens the lifetime of silica-based HPLC columns. If possible, organic buffers should be used at $\mathrm{pH}$ greater than 7.

- Ammonium bicarbonate buffers usually are prone to $\mathrm{pH}$ changes and are usually stable for only 24 to 48 hours. The $\mathrm{pH}$ of this mobile phase tends to become more basic due to the release of carbon dioxide.

- Ammonium salts are generally more soluble in organic/water mobile phases.

- Some salt buffers are hygroscopic. This may lead to changes in the chromatography (increased tailing of basic compounds, and possibly selectivity differences).

- Microbial growth can quickly occur in buffered mobile phases that contain little or no organic modifier. This growth will accumulate on column inlets and can damage chromatographic performance.

- After buffers are prepared, they should be filtered through a $0.2-\mu \mathrm{m}$ filter.

- Mobile phases should be degassed.

\subsection{Selection of detectors}

Detector is a very important part of HPLC. Selection of detector depends on the chemical nature of analyses, potential interference, limit of detection required, availability and/or cost of detector (Table 2).

Table 2: HPLC Detector Choice

Type of compound can be detected

Detector

Compounds with chromophores, such as aromatic rings or multiple alternating double bonds.

UV-Visible \&Photodiode array

Fluorescence detector

Conductivity detector

Electrochemical detector

Fluorescent compounds, usually with fused rings or highly conjugated planer system.

Charged compounds, such as inorganic ions and organic acid.

Refractive Index detector \& Evaporative light scattering Compounds that do not show characteristics usable by the other detectors, eg.polymers,

detector saccharides.

UV-visible detector is versatile, dual wavelength absorbance detector for HPLC. This detector offers the high sensitivity required for routine UV-based applications to low-level impurity identification and quantitative analysis. Photodiode Array (PDA) Detector offers advanced optical detection for Waters analytical HPLC, preparative HPLC, or LC/MS system solutions. Its integrated software and optics innovations deliver high chromatographic and spectral sensitivity. Refractive index chromatographic and spectral sensitivity, stability and reproducibility, which make this detector the ideal solution for analysis of components with limited or no UV absorption. Multiwavelength Fluorescence Detector offers high sensitivity and selectivity fluorescence detection for quantitating low concentrations of target compounds [31], [32].

\subsection{Preparation of sample solutions for method development}

The components being analyzed should be stable in solution (diluent). During initial method development, preparations of the solutions in amber flasks should be performed until it is determined that the active component is stable at room temperature and does not degrade under normal laboratory conditions. The sample solution should be filtered, the use of a 0.22 or $0.45 \mu \mathrm{m}$ pore-size filter is generally recommended for removal of particulates. Filtration is a preventive maintenance tool for HPLC analyses [31-34].

Sample preparation is a critical step of method development that the analyst must investigate. The effectiveness of the syringe filters is largely determined by their ability to remove contaminants/insoluble components without leaching undesirable artifacts into the filtrate. If any additional peaks are observed in the filtered samples, then the diluent must be filtered to determine if a leachable component is coming from the syringe filter housing/filter.

\subsection{Optimization method}

The experimental conditions should be optimized to get desired separations and sensitivity after getting appropriate separations. Stability indicating assay experimental conditions will be achieved through planned/systemic examination on parameters including $\mathrm{pH}$ (if ionic), mobile phase components and ratio, gradient, flow rate, sample amounts, Injection volume and diluents solvent type [35].

\section{Validation method}

Validation of an analytical procedure is the process by which it is established, by laboratory studies, that the performance characteristics of the procedure meet the requirements for its intended use. The methods validation process for analytical procedures begins with the planned and systematic collection by the applicant of the validation data to support analytical procedures. All analytical methods that are intended to be used for analyzing any clinical samples will need to be validated. The validation of analytical methods is done as per ICH guidelines [36].

The main typical analytical performance characterstics which may be tested during methods validation are System Suitability, Accuracy, Precision, Repeatability, Intermediate precision, Linearity, Detection limit, Quantification limit, Specificity, Range, Robustness, System suitability determination, Forced degradation studies and Solution stability studies.

\subsection{System suitability}


System suitability testing originally believed by the industry of pharmaceuticals to decide whether a chromatographic system is being utilized day today in a routine manner in pharmaceutical laboratories where quality of results is most important which is suitable for a definite analysis.

The parameters used in the system suitability tests (SST) report are: Number of theoretical plates or Efficiency (N), Capacity factor (K), Separation or Relative retention ( $\alpha$ ), Resolution (Rs), Tailing factor (T), Relative Standard Deviation (RSD) [37-40].

\subsubsection{Number of theoretical plates/efficiency $(\mathbf{N})$}

In a specified column, efficiency is defined as the measurement of the degree of peak dispersion and it should have the column characteristics. The efficiency is conveyed in terms of number of theoretical plates. The formula of calculation of $\mathrm{N}$ is illustrated bellow in the following

Sigma/tangential method (USP method) With the help of sigma/tangential method $\mathrm{N}$ is calculated which is shown in the following figure 2 duly noting the formula for calculation of $\mathrm{N}$.

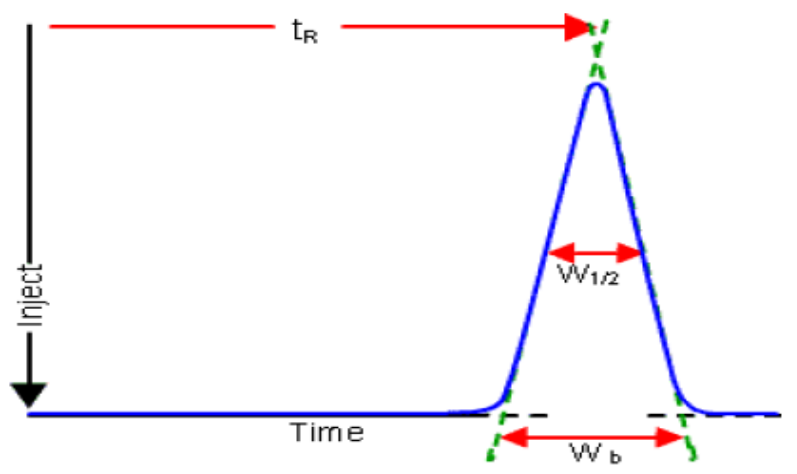

Fig. 2: Sigma/Tangential Method Relating to Determination of N.

$N=16\left(\frac{t_{R}}{W_{b}}\right)^{2}=5.54\left(\frac{t_{R}}{W_{\frac{1}{2}}}\right)^{2}$

$\mathrm{N}=$ Number of theoretical plates.

$t_{R}=$ Retention time or retention distance.

$\mathrm{h}=$ Peak height.

$\mathrm{w}_{\mathrm{b}}=$ width of the peak at the base line.

The plate number depends on column length. Theoretical plate number is the measure of column efficiency. As stated by plate theory, the analyte will be in instant equilibrium with stationary phase and column has to be divided into number of hypothetical plates and each plate consists of a fixed height and analyte spends finite time in the plate. Height equivalent to theoretical plate (HETP) is given by following formula:

$\mathrm{HETP}=\mathrm{L} / \mathrm{N}$,

Where, $\mathrm{L}=$ length of column. $\mathrm{N}=$ plate number

\subsubsection{Capacity ratio or capacity factor (K')}

The capacity factor (k') is a means of measuring the retention of an analyte on the chromatographic column.

$\mathbf{K}^{\prime}=\frac{\mathbf{t}_{\mathrm{R}}-\mathbf{t}_{\mathrm{M}}}{\mathbf{t}_{\mathrm{M}}}$

The above said capacity factor sometimes is called as a retention factor which has no dimension and independent from flow rate of mobile phase as well as column dimensions which is the measure of extent of retention relating to an analyte relative to an un-retained peak. Where tR implies retention time of the sample peak and retention time of an un-retained peak is $t_{\mathrm{M}} . \mathrm{k}^{\prime}=0$ means no compound is left in the column. Generally the value of $k^{\prime}$ is $>2$ [41].

\subsubsection{Relative retention or separation factor $(\alpha)$}

The selectivity (or separation) factor $(\alpha)$ is the ability of the chromatographic system to 'chemically' distinguish between sample components. 


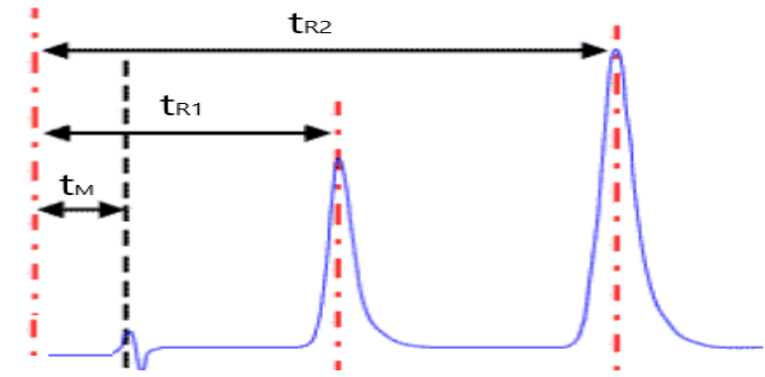

Inject

Fig. 3: Determination of Separation Factor (A).

$\alpha=\frac{\left(t_{R 2}-t_{M}\right)}{\left(t_{R 1}-t_{M}\right)}$

$t_{1}=$ the retention time from the point of injection of reference peak defined. (Suppose no reference peak is found, value would be zero). $\mathrm{t}_{2}=$ Retention time calculated from point of injection.

$\mathrm{t}_{\mathrm{M}}=$ Unretained peak time (Retention time $\left(\mathrm{t}_{\mathrm{R}}\right)$ of an inert component not retained by the column).

\subsubsection{Resolution (Rs)}

Resolution is the capability of the column to separate 2 components in 2 individual peaks or chromatographic zones and it is improved by enhancing column length, reduction of particle size and rising temperature, altering the eluent or stationary phase. It can be told in terms of ratio of separation of the apex of two peaks by the tangential width average of the peaks. By using the following formula resolution is calculated.

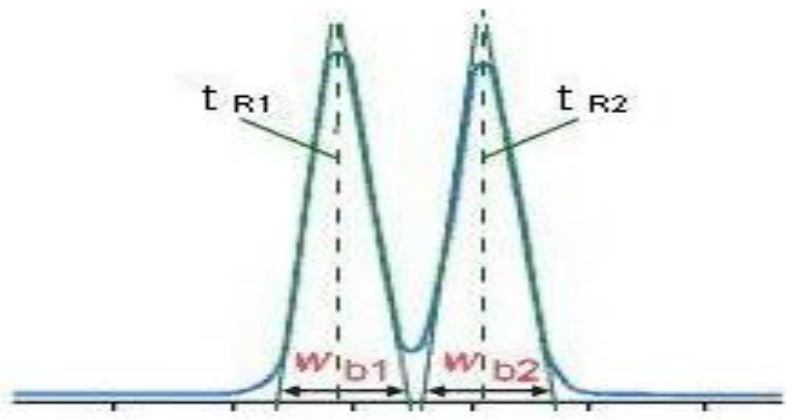

Fig. 4: Determination of Resolution between Two Peaks.

$$
R s=\frac{\left(t_{R 2}-t_{R 1}\right)}{\left(0.5\left(t_{w b 1}-t_{w b 2}\right)\right)}
$$

$t_{\mathrm{R} 1}$ and $t_{\mathrm{R} 2}$ are the retention times for the two peaks of components. $t_{\mathrm{wb} 1}$ and $t_{\mathrm{wb} 2}$ At the baseline lies between tangents drawn to the sides of the peaks. (Tangents are drawn at 0.6 times the peak height). If the peaks are correctly symmetric, provided the valley between the two peaks should touch the baseline Rs is 1.5 . Generally good value of resolution is Rs $\geq 2$ should be adequate and preferred normally [42].

\subsubsection{Resolution factor $(R)$}

Resolution is a function of capacity factor, function of selectivity and a function of efficiency (or) number of theoretical plates ( $\mathrm{N}$ ). In order to separate any two peaks you must have right capacity factor ideally between 2 and 10, but appropriate selectivity is required i.e., ideally 1.2 and enough efficiency i.e., number of theoretical plates (more than 2000 theoretical plates). Resolution should be $\geq 1.5$. 1.5 defines baseline resolution.

$$
R=\frac{1}{4} \sqrt{N}\left(\frac{\alpha-1}{\alpha}\right)\left(\frac{K^{\prime}}{K^{\prime}+1}\right)
$$

\subsubsection{Tailing factor or asymmetry factor}

Chromatographic peak assumed to have a Gaussian shape under ideal conditions. However in practical conditions, there is always a deviation from normal distribution which indicates non- uniform migration and non-uniform distribution process. The asymmetry factor and tailing factor are roughly same and rarely accurate and equal in most cases. Values should normally between 1.0-1.5 and values greater than 2 are unacceptable. The peak asymmetry is computed by utilizing the following formula [43].

$\mathrm{As}=\mathrm{B} / \mathrm{A}$

Where:

As $=$ peak asymmetry factor.

$\mathrm{B}=$ distance from the point at peak midpoint to the trailing edge (measured at $10 \%$ of peak height).

$\mathrm{A}=$ distance from the leading edge of peak to the midpoint. (Measured at $10 \%$ of peak height). 
Table 3: System Suitability Parameters are shown in the Following.

\begin{tabular}{ll}
\hline Parameter name & Acceptance criteria \\
\hline Number of theoretical plates or Efficiency (N) & $>2000$ \\
Capacity factor (K) & $<1$ \\
Separation or Relative retention $(\alpha)$ & $>1$ \\
Resolution (Rs) & $>1.5$ \\
Tailing factor or Asymmetry(T) & $<2$ \\
Relative Standard Deviation (RSD) & $<2$ \\
\hline
\end{tabular}

\subsection{Specificity}

Specificity is the ability to assess unequivocally the analyte in the presence of components which may be expected to be present. Typically these might include impurities, degradants, matrix, etc.

\subsection{Accuracy}

The accuracy of an analytical procedure expresses the closeness of agreement between the value which is accepted either as a conventional true value or an accepted reference value and the value found.

\subsection{Precision}

The precision of an analytical procedure expresses the closeness of agreement (degree of scatter) between a series of measurements obtained from multiple sampling of the same homogeneous sample under the prescribed conditions. Precision may be considered at three levels: repeatability, intermediate precision and reproducibility.

Precision should be investigated using homogeneous, authentic samples. However, if it is not possible to obtain a homogeneous sample it may be investigated using artificially prepared samples or a sample solution.

The precision of an analytical procedure is usually expressed as the variance, standard deviation or coefficient of variation of a series of measurements.

\subsection{Repeatability}

Repeatability expresses the precision under the same operating conditions over a short interval of time. Repeatability is also termed intraassay precision.

\subsection{Intermediate precision}

Intermediate precision expresses within-laboratories variations: different days, different analysts, different equipment, etc.

\subsection{Reproducibility}

Reproducibility expresses the precision between laboratories (collaborative studies, usually applied to standardization of methodology).

\subsection{Detection limit}

The detection limit of an individual analytical procedure is the lowest amount of analyte in a sample which can be detected but not necessarily quantified as an exact value.

\subsection{Quantification limit}

The quantification limit of an individual analytical procedure is the lowest amount of analyte in a sample which can be quantitatively determined with suitable precision and accuracy. The quantification limit is a parameter of quantitative assays for low levels of compounds in sample matrices, and is used particularly for the determination of impurities and/or degradation products.

\subsection{Linearity}

The linearity of an analytical procedure is its ability to obtain test results which are directly proportional to the concentration of analyte in the sample.

\subsection{Range}

The range of an analytical procedure is the interval between the upper and lower concentration of analyte in the sample for which it has been demonstrated that the analytical procedure has a suitable level of precision, accuracy and linearity.

\subsection{Robustness}

The robustness of an analytical procedure is a measure of its capacity to remain unaffected by small, but deliberate variations in method parameters and provides an indication of its reliability during normal usage.

\subsection{Forced degradation studies}


Forced degradation or stress studies are undertaken to deliberately degrade the sample. These studies are used to evaluate an analytical method's ability to measure an active ingredient and its degradation products, without interference, by generating potential degradation products. During validation of the method, the substance are exposed to acid, base, heat, light and oxidizing agent to produce approximately $10 \%$ to $30 \%$ degradation of active substance. The studies can also provide information about the degradation pathways and degradation products that could form during storage [44].

\subsection{Solution stability studies}

During validation the stability of standards and samples is established under normal conditions, normal storage conditions, and sometimes in the instrument to determine if special storage conditions are necessary, for instance, refrigeration or protection from light [45].

\section{Conclusion}

It can be concluded from the entire review that HPLC is a versatile, reproducible chromatographic technique for the estimation of each products. It has wide applications in different fields in term of quantitative and qualitative estimation of active molecules.

\section{References}

[1] Joseph C. Touchstone (2006), History of Chromatography, Journal of liquid chromatography, volume 8 (16), 1647-1665. https://doi.org/10.1080/10826079308021679.

[2] Sánchez J. L., Agnes I. B. L., Antonio S. K., Carretero S. (2018), Chromatographic Technique: High-Performance Liquid Chromatography (HPLC), Modern Techniques for Food Authentication (Second Edition).

[3] Ravisankar P., Anusha S., Kommanaboina S., Kumar U. A. (2019), Fundamental Chromatographic Parameters, Int. J. Pharm. Sci. Rev. Res, 55(2) $46-50$.

[4] Ravisankar P, Geethika S. A, Rachana G, Srinivasa B. P, Bhargavi J (2019), Bioanalytical method validation: A comprehensive review, Int. J. Pharm.Sci.Rev.Res., 56(1), 50-58.

[5] Neue U. D. (1997), Wiley Analytical Science, HPLC Columns: Theory, Technology, and Practice.

[6] Karatepe M. (2004), Simultaneous Determination of Ascorbic Acid and Free Malondialdehyde in Human Serum by HPLC-UV, LCGC NORTH AMERICA, (22). 362-365.

[7] Dinçer Z., Basan H., Göger N. G. (2003), Journal of Pharmaceutical and Biomedical Analysis, Quantitative determination of ambroxol in tablets by derivative UV spectrophotometric method and HPLC 5 (31), 867-872. https://doi.org/10.1016/S0731-7085(02)00664-7.

[8] Hamilton J.G., Comai K. (1988), Separation of neutral lipid, free fatty acid and phospholipid classes by normal phase HPLC. Lipids, (23). 11501153. https://doi.org/10.1007/BF02535282.

[9] Horvath C., Melander W. (1977), Liquid Chromatography with Hydrocarbonaceous Bonded Phases; Theory and Practice of Reversed Phase Chromatography, Journal of Chromatographic Science, 9 (15), 393-404, https://doi.org/10.1093/chromsci/15.9.393.

[10] Gallant S.R., Vunnum S., Cramer S.M. (1996), Optimization of preparative ion-exchange chromatography of proteins: linear gradient separations, Journal of Chromatography A, 2 (23), 295-314. https://doi.org/10.1016/0021-9673(95)00909-4.

[11] Oeyen E., Mol K. V., Baggerman G., Willems H., Boonen K., Rolfo C., Pauwels P. Jacobs A., Schildermans K., Cho W., Mertens. I. (2018), Ultrafiltration and size exclusion chromatography combined with asymmetrical-flow field-flow fractionation for the isolation and characterisation of extracellular vesicles from urine. Journal of Extracellular Vesicles, 1-7. https://doi.org/10.1080/20013078.2018.1490143.

[12] Malviya R, Bansal V, Pal O.P. and Sharma P.K. (2010), High Performance Liquid Chromatography: A Short Review, Journal of Global Pharma Technology 2(5), 22-26.

[13] Wiese S., Teutenberg T. and Schmidt T. C. (2011), A general strategy for performing temperature-programming in high performance liquid chromatography_Prediction of segmented temperature gradients, Journal of Chromatography A, 10. (39), 68986906. https://doi.org/10.1016/j.chroma.2011.08.022.

[14] Gupta V., Jain A. D. K., Gill.N.S, Gupta K. (2012), Development and validation of HPLC method - a review. Int. Res J Pharm. App Sci., 2 (4), 17. 25.

[15] Kaushal C, Srivastava B (2010), A Process of Method Development: A Chromatographic Approach, J Chem Pharm Res, 2(2), 519-545.

[16] Claessensa H.A., Stratena M.A., Kirklandb J.J. (1996), , Effect of buffers on silica-based column stability in reversed-phase high-performance liquid chromatography, Journal of Chromatography A, 1-2 (728), 259-270. https://doi.org/10.1016/0021-9673(95)00904-3.

[17] Dejanovic K. N. and Cabaniss S. E. (2004), Reverse-Phase HPLC Method for Measuring Polarity Distributions of Natural Organic Matter, J. environmental Science \& Technology 38 (4), 1108-1114. https://doi.org/10.1021/es0344157.

[18] Veigure R., Lossmann K., Hecht M., Parman E., Born R., Leito I., Herodes K. ( 2020), Kipper K., Retention of acidic and basic analytes in reversed phase column using fluorinated and novel eluent additives for liquid chromatography-tandem mass spectrometry, Journal of Chromatography A,(1613), 460-667. https://doi.org/10.1016/j.chroma.2019.460667.

[19] Dalluge J. J., Nelson B. C., Jeanice M. Thomas B., Sander L.C. (1998), Selection of Column and Gradient Elution System for the Separation of Catechins in Green Tea Using High-Performance Liquid Chromatography, Journal of Chromatography A 793 (2), $265-274$. https://doi.org/10.1016/S0021-9673(97)00906-0.

[20] Villiersa A., Cabooter D., Lynen F., Desmet G., Sandrac P. (2009), High performance liquid chromatography analysis of wine anthocyanins revisited: Effect of particle size and temperature, Journal of Chromatography A, 1216 (15), 3270-3279. https://doi.org/10.1016/j.chroma.2009.02.038

[21] Noman A, Bukhaiti ALWedad Q, Alfarga A,Abed Sherif M, Mahdi AA. And Waleed AA. (2016), HPLC technique used in food analysis-Review. International Journal of Agriculture Innovations and Research. 5(2), 181-188.

[22] Snyder LR, Kirkland JJ, Dolan JW (2011). Introduction to modern liquid chromatography. John Wiley \& Sons. New York.

[23] Xiang Y, Liu Y, Lee ML (2006). Ultrahigh pressure liquid chromatography using elevated temperature. Journal of Chromatography. 1104 (1), 198 202. https://doi.org/10.1016/j.chroma.2005.11.118.

[24] Horvath CG, Preiss BA, Lipsky SR. (1967) fast liquid chromatography. Investigation of operating parameters and the separation of nucleotides on pellicular ion exchangers. Analytical chemistry, 39 (12), 1422-1428. https://doi.org/10.1021/ac60256a003.

[25] Kumar S. D and Kumar D.R.H. (2013) Importance of RP-HPLC in Analytical method development: A review. International journal of novel trends in pharmaceutical sciences; 3 (1) 15-23.

[26] Chandra M. (2013), Buffers, A guide for the preparation and use of buffers in biological system: available from: www.calbiochem.com. Accessed April 05, 2013.

[27] Lindholm J. (2004) Development and Validation of HPLC method for Analytical and Preparative Purpose. Acta Universities Upsaliensis Uppsalapp. 13-14.

[28] Dolan (2015) HPLC Solutions \#53: Temperature and Retention. J. Separation Science. [Online] https://owl. english.purdue.edu/owl/resource/747/08/ (accessed Jan 27, 2015). 
[29] Snyder LR, Kirkland JJ, Glach JL. (1997) Practical HPLC Method Development, 2nd edition. New York. John Wiley \&Sons pp. $233-291$. https://doi.org/10.1002/9781118592014.

[30] Patwekar SL, Sakhare RS and Nalbalwar N. N. (2015), HPLC method development and validation- A general Concept, International Journal of Chemical and Pharmaceutical Sciences, 6 (1) 8-14.

[31] British Pharmacopoeia. (International ed.) 1993; Vol. 1:429, 483. Published on the Recommendation of the Medicines Commissions Pursuant to Medicines Act 1968, 1993

[32] United States Pharmacopoeia 29 NF 24, Published on the Recommendation of the Medicines Commissions Pursuant to Medicines, page no. 587

[33] Skoog, West, Holler, Crouch, "Fundamentals of analytical chemistry", eighth edition, 2009 (Indian edition), c engage learning India pvt ltd, New delhi, pp. 271-280.

[34] Kasture A.V, Mahadik K.R, Wadodkar S.G, More H.N., "A textbook of pharmaceutical analysis, Instrumental methods”, Nirali Prakashan, vol.2, 9th edition, pp. 5-7, 28-30.

[35] Outinen K., Vuorela H., Hiltunen R. (1996), Optimization of HPLC separation by computer-assisted optimization methods Comparison of three methods, European Journal of Pharmaceutical Sciences, (4) 199-210. https://doi.org/10.1016/0928-0987(95)00040-2.

[36] ICH Topic Q 2 (R1) Validation of Analytical Procedures: Text and Methodology.

[37] Barnett K. L., Harrington B., Graul T. W. (2017) Validation of liquid chromatographic methods. Liquid Chromatography, 533-552. https://doi.org/10.1016/B978-0-12-805393-5.00022-1.

[38] Barnett K.L., Harrington B., Graul T.W. (2013), Validation of Liquid Chromatographic Methods, Liquid Chromatography 57-73. https://doi.org/10.1016/B978-0-12-415806-1.00003-6.

[39] Bretnall A. E., Clarke G. S (2011) Validation of Analytical Test Methods. Handbook of Modern Pharmaceutical Analysis, $429-457$. https://doi.org/10.1016/B978-0-12-375680-0.00011-5.

[40] Jimidar M. I., Heylen P., De Smet M. (2007), HPLC Method Development for Pharmaceuticals, Method validation, (16) 441-458. https://doi.org/10.1016/S0149-6395(07)80022-5.

[41] Lee, Y., So, M.S., Lee, J.W. (1996), Korean Retention models of capacity factor with different compositions of organic modifier in RP-HPLC. J. Chem. Eng., (13) 578-584. https://doi.org/10.1007/BF02706024.

[42] West S. D. (1987) The Prediction of Reversed-Phase HPLC Retention Indices and Resolution as a Function of Solvent Strength and Selectivity, Journal of Chromatographic Science, 3 (25), 122-129, https://doi.org/10.1093/chromsci/25.3.122.

[43] Song P. D. and Wang J. (2003) Modified resolution factor for asymmetrical peaks in chromatographic separation, Journal of Pharmaceutical and Biomedical Analysis,32 (6) 1105-1112. https://doi.org/10.1016/S0731-7085(03)00026-8.

[44] Reynolds DW, Facchine KL, Mullaney JF, Alsante KM, Hatajik TD, Motto MG. (2002) Available guidance and best practices for conducting forced degradation studies. Pharm Tech., 48-56.

[45] Shao Y., Alluri R., Mummert M., Koetter U. and Lech S. (2004) A stability-indicating HPLC method for the determination of glucosamine in pharmaceutical formulations, Journal of Pharmaceutical and Biomedical Analysis, 35 (3) 625-631. https://doi.org/10.1016/j.jpba.2004.01.021. 